\title{
Radial nerve palsy associated with closed humeral shaft fractures: a systematic review of 1758 patients
}

\author{
Laurent A. M. Hendrickx ${ }^{1,2} \cdot$ Nick F. J. Hilgersom $^{1,3} \cdot$ Hassanin Alkaduhimi $^{3} \cdot$ Job N. Doornberg ${ }^{1,2}$. \\ Michel P. J. van den Bekerom ${ }^{3}$
}

Received: 20 January 2020 / Published online: 13 April 2020

(c) The Author(s) 2020

\begin{abstract}
Background and purpose Humeral shaft fractures are often associated with radial nerve palsy (RNP) (8-16\%). The primary aim of this systematic review was to assess the incidence of primary and secondary RNP in closed humeral shaft fractures. The secondary aim was to compare the recovery rate of primary RNP and the incidence of secondary RNP between operative and non-operative treatment.

Methods A systematic literature search was performed in 'Trip Database', 'Embase' and 'PubMed' to identify original studies reporting on RNP in closed humeral shaft fractures. The Coleman Methodology Score was used to grade the quality of the studies. The incidence and recovery of RNP, fracture characteristics and treatment characteristics were extracted. Chi-square and Fisher exact tests were used to compare operative versus non-operative treatment.

Results Forty studies reporting on 1758 patients with closed humeral shaft fractures were included. The incidence of primary RNP was $10 \%$. There was no difference in the recovery rate of primary RNP when comparing operative treatment with radial nerve exploration (98\%) versus non-operative treatment $(91 \%)(p=0.29)$. The incidence of secondary RNP after operative and non-operative treatment was $4 \%$ and $0.4 \%$, respectively $(p<0.01)$.

Interpretation One-in-ten patients with a closed humeral shaft fracture has an associated primary RNP, of which $>90 \%$ recovers without the need of (re-)intervention. No beneficial effect of early exploration on the recovery of primary RNP could be demonstrated when comparing patients managed non-operatively with those explored early. Patients managed operatively for closed humeral shaft fractures have a higher risk of developing secondary RNP.
\end{abstract}

Level of evidence Level IV; Systematic Review.

Keywords Humerus $\cdot$ Radial nerve $\cdot$ Humeral shaft fracture $\cdot$ Fracture $\cdot$ Nerve palsy $\cdot$ Nerve injury

Electronic supplementary material The online version of this article (https://doi.org/10.1007/s00402-020-03446-y) contains supplementary material, which is available to authorized users.

Laurent A. M. Hendrickx ranhendrickx@gmail.com

1 Department of Orthopaedic Surgery, Amsterdam University Medical Centre, University of Amsterdam, Amsterdam Movement Sciences, Amsterdam, The Netherlands

2 Department of Orthopaedic and Trauma Surgery, Flinders Medical Centre, Flinders University, Adelaide 5042, Australia

3 Department of Orthopaedic Surgery, Onze Lieve Vrouwe Gasthuis, 1091 Amsterdam, The Netherlands

\section{Introduction}

Humeral shaft fractures account for 1-3\% of all fractures [1]. The incidence, 14 per 100,000 per year [1, 2], has increased over $100 \%$ over the past 25 years [3]. Due to its course around the humerus, the radial nerve is prone to injury in these fractures: radial nerve palsy (RNP) has been reported in $8-16 \%$ of humeral shaft fractures $[1,4,5]$.

Radial nerve palsy can either be primary or secondary. Primary RNP occurs at the onset of the fracture and may be caused by compression within the fracture site, transection by fracture fragments, or local pressure by swollen tissue. Secondary, or iatrogenic, RNP develops during the course of treatment. Secondary RNPs account for 10-20\% of all RNPs associated with humeral shaft fractures [6]. 
Though frequently seen, it remains unclear what the best treatment strategy for RNP associated with humeral shaft fractures is. Some recommend conservative management based on the experience that RNP often resolves spontaneously. A primary RNP following a closed humeral shaft fracture has been reported to recover in $>70 \%[4,6,7]$. Others suggest surgical management. They state early exploration can aid in diagnosing the type of RNP (e.g., neuropraxia) and may be associated with a reduced risk of nerve entrapment by scar tissue or exuberant callus [4, 6]. Early exploration also gives the opportunity to repair a lacerated nerve at an early stage, which may result in better outcome [6].

The most recent review investigating RNP in humeral shaft fractures dates to 2013 [5]; however, over the last 6 years 20 studies have been added to potentially contribute to our understanding [8-27]. Therefore, the primary aim of this study was to systematically review all contemporary evidence to assess the incidence and recovery rate of primary and secondary RNP in closed humeral shaft fractures. The secondary aim was to compare the recovery rate of primary RNP and the incidence of secondary RNP between operative and non-operative treatment.

\section{Materials and methods}

\section{Protocol}

This study adhered to the Preferred Reporting Items for Systematic Reviews and Meta-Analyses (PRISMA) guidelines
[28]. A study protocol was created prior to commencement of the study. The protocol was not registered.

\section{Selection criteria}

All original studies that assessed the outcome of operative or non-operative treatment of traumatic humeral shaft fractures were included. Studies had to report on the presence or absence of primary or secondary RNP to be included. Only studies including at least ten adult (i.e., $\geq 18$ years) patients with closed, non-pathological humeral shaft fractures were included. Studies that reported solely on patients with RNP were excluded to avoid selection bias. The inclusion and exclusion criteria are summarized in Table 1.

\section{Literature search strategy}

In collaboration with a clinical librarian 'Trip database', 'PubMed' and 'Embase' were searched to gather all available evidence on primary and secondary RNP associated with closed humeral shaft fractures. Details of the searches are displayed in Table 2. Searches were limited to English, German and Dutch papers, published since 1990. Searches were updated until 11 June 2019. Reference lists of included studies were manually searched to assure no studies meeting inclusion criteria were missed.

\section{Screening for eligibility}

Two authors ( $\mathrm{LH}$ and $\mathrm{NH}$ ) independently screened the title, abstracts and full texts of the studies for eligibility based on
Table 1 Inclusion and exclusion criteria

\begin{tabular}{ll}
\hline Inclusion criteria & Exclusion criteria \\
\hline $\begin{array}{ll}\text { Studies including } \geq 10 \text { adult }(\geq 18 \text { years) patients } \\
\text { with closed, traumatic humeral shaft fractures }\end{array}$ & Studies not reporting on the presence or absence of RNP \\
Cohort studies, case series, RCTs & Studies not reporting on the length of follow-up \\
& Open fracture, pathological fractures, non-union \\
\hline
\end{tabular}

Table 2 Literature search PubMed, Embase and Trip

\begin{tabular}{ll}
\hline Database & Search terms \\
\hline PubMed & $($ "Humerus"[Mesh] OR Humer*) \\
& AND \\
& $($ ("Radial Nerve"[Mesh]) OR (Radial AND (nerve OR nerves OR nervus OR nervous OR neuropathy OR \\
& palsy OR palsies OR paralys*))) \\
& AND \\
& $((" F r a c t u r e s$, Bone" $[$ Mesh]) OR Fractur*) \\
& humer* \\
& AND \\
& $($ radial AND (nerve OR nerves OR nervus OR nervous OR neuropathy OR palsy OR palsies OR paralys*)) \\
& AND \\
& fractur*
\end{tabular}


predetermined inclusion- and exclusion criteria. Disagreement was resolved by discussion. If no consensus could be reached, a senior author $(\mathrm{MB})$ gave the final verdict.

\section{Assessment of quality}

Two authors ( $\mathrm{LH}$ and $\mathrm{NH}$ ) independently assessed the quality of the studies using the 'Coleman Methodology Score' [29]. The 'Coleman Methodology Score' was adjusted to make it more suitable for the studies in the systematic review (Appendix A). The total score corresponded to either a poor (0-49), fair (50-69), good (70-84), or excellent (85-100) quality of the study. Mismatches in assigned scores were discussed by two authors ( $\mathrm{LH}$ and $\mathrm{NH}$ ). If no agreement could be reached, a senior author made the final decision (MB).

\section{Data extraction}

The following data were extracted from the included studies by one author ( $\mathrm{LH}$ ) and validated by a second author $(\mathrm{NH})$ : study characteristics, study design, patient characteristics, fracture characteristics, primary RNP, secondary RNP, recovery of RNP, time to recovery of RNP, type of treatment and type of approach used. Surgical approaches were categorized into three groups: (1) anterolateral (i.e. minimally invasive anterior, open anterolateral and open extended deltopectoral); (2) posterior (i.e., open posterior and minimally invasive posterior); and (3) lateral (i.e., lateral open). A more detailed description of the extracted data is displayed in the appendix (Appendix B).

\section{Statistical analysis}

Results were summarized as absolute numbers with percentages. Patients with primary radial neve palsy were not included in the analysis of the incidence of secondary RNP. Fisher's exact tests and Chi-square tests were used to compare the recovery rate of primary RNP and the incidence of secondary RNP in operative versus non-operative treatment of closed humeral shaft fractures.

\section{Results}

\section{Study selection}

1052 unique studies were identified, of which 37 met the inclusion criteria. Three additional studies were identified through cross-referencing, resulting in the final inclusion of 40 studies [8-27, 30-49]. The flowchart of the selection process is displayed in Fig. 1.

\section{Critical appraisal}

Median Coleman score was 56 (range 22-91), indicating a fair overall quality of included studies. 12 studies were ranked poor [9-11, 21, 34-39, 47, 49], 24 studies were ranked fair $[8,12,14-18,20,22-27,30-33,40,41,43,45$, $46,48], 3$ studies were ranked good [13, 42, 44] and 1 study was ranked excellent [19].

\section{Study characteristics}

The 40 included studies described 1758 patients with closed humeral shaft fractures. Study sizes ranged from 10 to 212 patients. Follow-up varied from 3 to 75 months.

There were four randomized controlled trials (RCTs) [13, $19,41,42]$, six prospective studies $[8,12,43-46]$ and 30 retrospective studies [9-11, 14-18, 20-27, 30-40, 47-49]. One study was written in German [49], and the remaining studies were written in English. 24 studies were conducted in Asia $[11-13,15-18,20,22-25,27,30,31,33,36-38$, 40-42, 44, 46, 48], six in Europe [21, 32, 39, 45, 47, 49], five in South-America [14, 19, 26, 34, 43], three in NorthAmerica [9, 10, 35] and one in Africa [8].

Two studies reported solely on the effect of non-operative management [20,45], five studies reported on the effect of operative and non-operative management $[9,10,19,35,47]$ and the remaining studies reported on the effect of one or more surgical interventions $[8,11-18,21-27,30-34,36-46$, 48, 49]. Study characteristics are displayed in Appendix C.

\section{Incidence primary RNP}

22 studies reported on primary RNP $[9-12,14,16,18,20$, $22,23,26,33-35,37-40,43,45,47,49]$. These studies consisted of 922 patients of which 88 presented with primary RNP (10\%) (Table 3).

Primary RNP was reported in 0 out of 17 proximal 1/3rd, 5 out of 67 middle $1 / 3$ rd and 19 out of 152 (13\%) distal 1/3rd humeral shaft fractures (Table 4).

Primary RNP was seen in 10 out of 62 spiral, 3 out of 25 oblique, 2 out of 47 transverse and 5 out of 97 comminuted humeral shaft fractures.

\section{Recovery of primary RNP}

The overall recovery rate of primary RNP was $94 \%$ (Table 3). The longest time to full spontaneous recovery of primary RNP reported was 12 months [10].

The recovery rate of primary RNP in non-operatively managed humeral shaft fractures was $91 \%$. In 21 out of 23 cases the radial nerve showed full recovery without reintervention. Definitive management of the patients without recovery of primary RNP was not reported [10, 20]. 
Fig. 1 Flow chart of study selection process

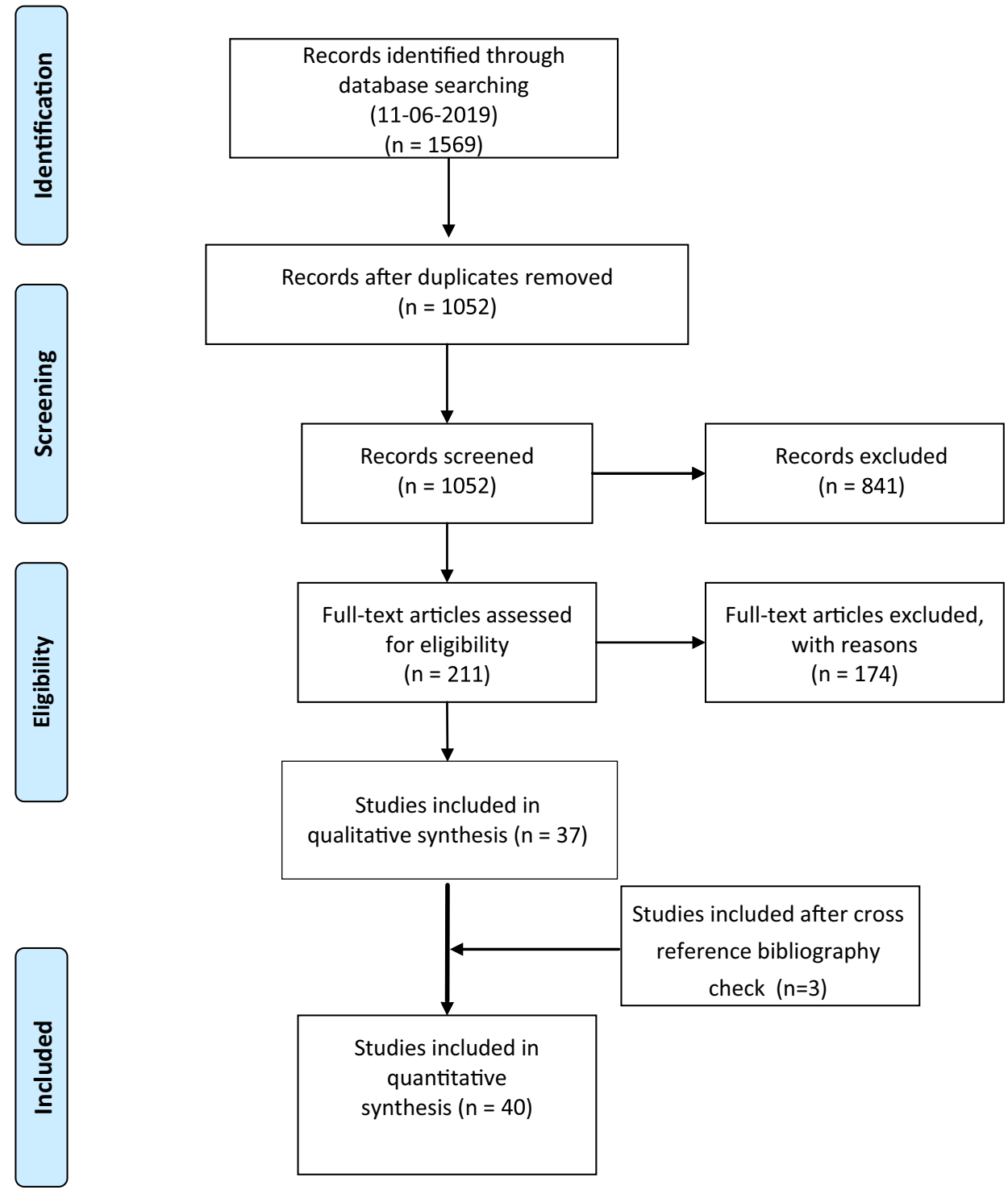

Table 3 Incidence and recovery rate of primary radial nerve palsy in closed humeral shaft fractures

\begin{tabular}{lllll}
\hline & $\begin{array}{l}\text { Overall } \\
N=922^{\mathrm{a}}\end{array}$ & $\begin{array}{l}\text { Non-operative } \\
N=240\end{array}$ & $\begin{array}{l}\text { Operative } \\
N=682\end{array}$ & $p$ value \\
\hline Primary RNP, $n(\%)$ & $88(10 \%)$ & $23(10 \%)$ & $65(10 \%)$ & \\
$\begin{array}{l}\text { Recovery without } \\
\text { re-intervention, } \\
n(\%)\end{array}$ & $83(94 \%)$ & $21(91 \%)$ & $62(95 \%)$ & 0.60 \\
\hline
\end{tabular}

${ }^{a} 18$ studies $(n=836)$ excluded patients with primary radial nerve palsy and are not included in this analysis

The recovery rate of primary RNP in operatively treated humeral shaft fractures was $95 \% .62$ out of 65 primary RNPs showed full recovery without reintervention. In 41 of these 65 , the radial nerve was explored during surgery, of which $40(98 \%)$ showed full recovery. One of three patients not
Table 4 Fracture characteristics and primary radial nerve palsy

\begin{tabular}{lrl}
\hline & $N$ & Primary RNP \\
\hline Fracture location & 17 & \\
Proximal $1 / 3$ & 67 & 5 \\
Middle $1 / 3$ & 152 & $19(13 \%)$ \\
Distal $1 / 3$ & & \\
Fracture type & 62 & 10 \\
Spiral & 25 & 3 \\
Oblique & 47 & 2 \\
Transverse & 97 & 5 \\
Comminuted & &
\end{tabular}

recovering from primary RNP after initial operative treatment was managed with a tendon transfer after 6-months [35], another patient underwent radial nerve exploration 
after 3 months during which the radial nerve was released from callus [37]. In the third patient, definitive management was not reported [10].

There was no significant difference in the recovery rate of primary RNP for the initial operative or non-operative treatment of closed humeral shaft fractures $(p=0.60)$, nor was there a difference when operative treatment with nerve exploration was compared to non-operative treatment $(p=0.29)$.

\section{Incidence of secondary RNP}

All studies reported on secondary RNP. In 57 out of 1670 (3\%) patients, treatment was complicated by secondary RNP (Table 5) The incidence of secondary RNP in nonoperatively treated patients was $0.4 \%$, while the incidence of secondary RNP in operatively treated patients was $4 \%$. This difference was statistically significant $(p<0.01)$.

\section{Fixation technique and secondary radial nerve palsy}

11 studies [11-13, 15, 21, 30, 33, 37, 38, 42, 49] assessed the outcome of intramedullary nailing, describing a total of 385 patients. In seven patients (2\%), treatment was complicated by secondary RNP (Table 6 ).

17 studies [8, 14, 17, 19, 24, 26, 27, 30-32, 34, 36, 39, $43,44,46,48]$ reported on the effect of minimally invasive plate osteosynthesis, consisting of 436 patients. In 12 patients (3\%), treatment was complicated by secondary RNP.

13 studies [13, 16-18, 22, 23, 25, 33, 35, 40-42, 48] reported on 553 patients treated with conventional plating. In 36 patients (7\%) treatment was complicated by secondary RNP.

\section{Surgical approach and secondary RNP}

In 14 out of 467 patients (3\%) in whom an anterolateral approach was used [17-19, 22, 24, 26, 27, 30-32, 34, 36, $39,43,44,46,48]$ and in 17 out of $356(5 \%)$ patients in whom a posterior approach was used [8, 14, 23, 25, 40, 41], treatment was complicated by secondary RNP (Table 6 ). In none of the 46 patients in whom a lateral approach was used secondary RNP occurred [16, 25].

\section{Recovery from secondary RNP}

21 studies [8, 10-15, 17, 19, 21-23, 25, 27, 33, 35, 36, 40, $42,44,48]$, with a weighted mean follow-up of 19 months, described the recovery from secondary RNP. 51 out of 54 patients with secondary RNPs recovered without reintervention (94\%) (Table 5). The longest time to spontaneous recovery of secondary RNP reported was 12 months [48]. In three patients, secondary RNP did not resolve without reintervention [35, 44]. One patient was initially managed nonoperatively and went on to conventional plating 2 months later because of unresolved secondary RNP and malalignment. The radial nerve was found to be intact and recovered after surgery [35]. The other two patients were managed operatively. One developed secondary RNP immediately after minimally invasive plate osteosynthesis. This patient was re-operated and underwent surgical exploration with plate reapplication after which the RNP resolved within $48 \mathrm{~h}$ [44]. The other patient was initially managed operatively with conventional plating. In this patient, the radial nerve showed no recovery at final follow-up 3 months after surgery [35].

Table 6 Surgical technique and incidence of secondary radial nerve palsy

\begin{tabular}{lll}
\hline & $\begin{array}{l}\text { Total patients } \\
\text { treated } \\
N^{\mathrm{a}}\end{array}$ & $\begin{array}{l}\text { Secondary RNP } \\
n(\%)\end{array}$ \\
\hline $\begin{array}{l}\text { Fixation technique } \\
\text { IMN }\end{array}$ & 385 & $7(2 \%)$ \\
Conventional plating & 553 & $36(7 \%)$ \\
MIPO & 436 & $12(3 \%)$ \\
Surgical approach & & $14(3 \%)$ \\
Anterolateral approach & 467 & $17(5 \%)$ \\
Posterior approach & 356 & 0 \\
Lateral approach & 46 & \\
\hline
\end{tabular}

${ }^{\text {a }}$ Patients with primary RNP were not included in this analysis
Table 5 Incidence and recovery rate of secondary radial nerve palsy in closed humeral shaft fractures

\begin{tabular}{|c|c|c|c|c|}
\hline & Overall $N=1670^{\mathrm{a}}$ & $\begin{array}{l}\text { Non-operative } \\
N=269^{\text {a }}\end{array}$ & $\begin{array}{l}\text { Operative } \\
N=1401^{\text {a }}\end{array}$ & $p$ value \\
\hline Secondary RNP, $n(\%)$ & $57(4 \%)$ & $1(0.4 \%)$ & $56(4 \%)$ & $<0.01 *$ \\
\hline $\begin{array}{l}\text { Recovery without reinter- } \\
\text { vention, } n(\%)\end{array}$ & $51 / 54(94 \%)^{\mathrm{b}}$ & $0(0 \%)$ & $51 / 53(96 \%)^{\mathrm{b}}$ & \\
\hline
\end{tabular}




\section{Discussion}

One-in-ten patients with a closed humeral shaft fracture has an associated primary RNP, of which more than $90 \%$ recovers without the need of any (re-)intervention. No beneficial effect of early exploration on the recovery of primary RNP could be demonstrated when comparing patients managed non-operatively with those explored early. Moreover, patients managed operatively for a closed humeral shaft fracture had a significantly higher risk of developing secondary RNP.

The previously described findings should be appreciated with several limitations in mind. Firstly, the overall methodological quality of the studies was fair and included only three RCTs, while it furthermore consisted of observational studies. Although previous studies have also used this methodology of combining both RCT's and observational trials $[50,51]$, it is important to note that the risk of bias is substantially larger in observational studies. Secondly, the heterogeneity of the used interventions between included studies was high. Due to these limitations (i.e., limited methodological quality and heterogeneity), a proper meta-analysis could not be conducted, and results of this study should therefore not be interpreted as such. However, we do believe that this study may provide sufficient evidence to compare non-operative versus operative treatment, as it is difficult to conduct a study of higher methodological quality such as an RCT or meta-analysis. The former is difficult to conduct due to the low incidence of RNP, while the latter is not possible due to the absence of high-quality studies. The strengths of this systematic review include: (1) a comprehensive search of the literature in collaboration with a clinical librarian; (2) selection of studies and grading of the evidence by two authors independently; and (3) large number of studies included resulting in the largest series of closed humeral shaft fractures to date. Given these strengths, we believe that the reported incidence for primary and secondary RNP are accurate estimates of the true incidence in patients with closed humeral shaft fractures.

The incidence of primary RNP associated with closed humeral shaft fractures observed in this systematic review was $10 \%$. Previous studies have reported on incidences ranging from 8 to $12 \%[1,4,52]$. However, these studies included patients with open fractures $[1,4,52]$, pathological fractures [1], or were based on smaller numbers [1, 52].

In the current study, the radial nerve appeared to be particularly at risk in middle (7\%) and distal third (13\%) humeral shaft fractures as well as in spiral-type fractures $(16 \%)$. This is in line with previous studies reporting increased risks of primary RNP for these specific type of fractures $[1,4,5,53]$.
The overall recovery rate of primary RNP was $94 \%$. No significant difference in the recovery rate could be demonstrated when comparing non-operative treatment versus operative treatment with nerve exploration. We therefore support earlier recommendations $[4,54]$ to manage patients with a closed humeral shaft fracture and an associated RNP non-operatively if the fracture allows it. This avoids the risk of potential operative complications and does not affect the recovery of the radial nerve adversely, while high union rates have been demonstrated with non-operative management in closed humeral shaft fractures [55].

The current study demonstrated a significantly lower incidence of secondary RNP in patients who were treated non-operatively for closed humeral shaft fractures $(0.4 \%)$ compared to patients who received operative treatment (4\%). Other large series assessing secondary RNP after operative treatment in open and closed humeral shaft fractures report incidences ranging from 6 to $7 \%[24,53,56]$. This may advocate non-operative treatment in closed humeral shaft fractures if the fracture is expected to heal well with nonoperative treatment.

Although the use of ultrasound as a diagnostic modality in patients with RNP after humeral shaft fractures has become more common over the years, it is currently not part of the standard diagnostic workup. Bodner and colleagues were the first to describe the use of ultrasound in patients with RNP after humerus shaft fractures [57, 58]. In a prospective study including 11 patients, they correctly diagnosed the type of RNP based on their pre-operative ultrasound findings in all five patients who underwent nerve exploration [58]. A more recent study further highlights the potential of ultrasound in diagnosing RNP in humeral shaft fractures: in 11 out 12 patients undergoing radial nerve exploration, the pre-operative ultrasound findings were confirmed intraoperatively [59]. It is important to note that for both studies, it cannot be confirmed whether ultrasound findings in patients not undergoing nerve exploration were also correct $[58,59]$. This limitation was overcome in a cadaveric study by Cartwright et al., demonstrating the ability of ultrasound to diagnose nerve transection with a sensitivity and specificity of $89 \%$ and $95 \%$, respectively [60]. Given its non-invasive nature and its supposed accurate ability to diagnose and differentiate between various types of nerve injury, further research on the use of ultrasound in the diagnostic workup of RNP in humeral shaft fractures is merited.

In conclusion, this study demonstrated that one-in-ten patients with a closed humeral shaft fracture has an associated primary RNP. No significant difference in the recovery rate of primary RNP could be demonstrated when comparing groups which were initially managed non-operatively with those explored early. This suggests that that non-operative treatment does not affect the extent of nerve recovery adversely. Non-operative treatment of closed humeral shaft 
fractures is furthermore associated with a significantly lower risk of secondary RNP.

Acknowledgements We would like to thank and acknowledge Bert Berenschot for his assistance in the design and conceptualization of a comprehensive literature search.

Author contributions MPJB, LAMH: Study design; NFJH, LAMH: literature search; NFJH, LAMH, MPJB: screening for eligibility; NFJH, LAMH: data collection; NFJH, HA, LAMH: critical appraisal; NFJH, HA, LAMH: data analysis; MPJB, LAMH, JND, NFJH: writing manuscript; MPJB, JND, NFJH, HA: critical revision manuscript.

Funding The authors, their immediate relatives, and any research foundations with which they are affiliated have not received any financial payments or other benefits from any commercial entity related to the subject of this article.

\section{Compliance with ethical standards}

Conflict of interest The authors declare that they have no conflict of interest.

Ethical approval This article does not contain any studies with human participants or animals performed by any of the authors.

Open Access This article is licensed under a Creative Commons Attribution 4.0 International License, which permits use, sharing, adaptation, distribution and reproduction in any medium or format, as long as you give appropriate credit to the original author(s) and the source, provide a link to the Creative Commons licence, and indicate if changes were made. The images or other third party material in this article are included in the article's Creative Commons licence, unless indicated otherwise in a credit line to the material. If material is not included in the article's Creative Commons licence and your intended use is not permitted by statutory regulation or exceeds the permitted use, you will need to obtain permission directly from the copyright holder. To view a copy of this licence, visit http://creativecommons.org/licenses/by/4.0/.

\section{References}

1. Ekholm R, Adami J, Tidermark J et al (2006) Fractures of the shaft of the humerus. An epidemiological study of 401 fractures. J Bone Joint Surg Br 88:1469-1473

2. Bergdahl C, Ekholm C, Wennergren D et al (2016) Epidemiology and patho-anatomical pattern of 2,011 humeral fractures: data from the Swedish Fracture Register. BMC Musculoskelet Disord 17:159

3. Mahabier KC, Hartog DD, Van Veldhuizen J et al (2015) Trends in incidence rate, health care consumption, and costs for patients admitted with a humeral fracture in The Netherlands between 1986 and 2012. Injury 46:1930-1937

4. Shao YC, Harwood P, Grotz MRW et al (2005) Radial nerve palsy associated with fractures of the shaft of the humerus: a systematic review. J Bone Joint Surg Br 87:1647-1652

5. Li Y, Ning G, Wu Q et al (2013) Review of literature of radial nerve injuries associated with humeral fractures-an integrated management strategy. PLoS ONE 8:e78576

6. DeFranco MJ, Lawton JN (2006) Radial nerve injuries associated with humeral fractures. J Hand Surg Am 31:655-663
7. Chang G, Ilyas AM (2018) Radial nerve palsy after humeral shaft fractures: the case for early exploration and a new classification to guide treatment and prognosis. Hand Clin 34:105-112

8. Balam KM, Zahrany AS (2014) Posterior percutaneous plating of the humerus. Eur J Orthop Surg Traumatol 24:763-768

9. Belayneh R, Lott A, Haglin J et al (2019) Final outcomes of radial nerve palsy associated with humeral shaft fracture and nonunion. J Orthop Traumatol 20:18

10. Dielwart C, Harmer L, Thompson J et al (2017) Management of closed diaphyseal humerus fractures in patients with injury severity score $\geq 17$. J Orthop Trauma 31:220-224

11. Duygun F, Aldemir C (2017) Is locked compressive intramedullary nailing for adult humerus shaft fractures advantageous? Eklem Hastalik Cerrahisi 28:80-86

12. Ebrahimpour A, Najafi A, Manafi Raci A (2015) Outcome assessment of operative treatment of humeral shaft fractures by antegrade unreamed humeral nailing (UHN). Indian J Surg 77:186-190

13. Fan Y, Li Y-W, Zhang H-B et al (2015) Management of humeral shaft fractures with intramedullary interlocking nail versus locking compression plate. Orthopedics 38:e825-e829

14. Gallucci GL, Boretto JG, Alfie VA et al (2015) Posterior minimally invasive plate osteosynthesis (MIPO) of distal third humeral shaft fractures with segmental isolation of the radial nerve. Chir Main 34:221-226

15. Han KJ, Lee DH, Bang JY (2017) Do Cerclage cables delay the time to bone union in patients with an unstable humeral shaft fracture treated with intramedullary nails? Yonsei Med J 58:837-841

16. Kharbanda Y, Tanwar YS, Srivastava V et al (2017) Retrospective analysis of extra-articular distal humerus shaft fractures treated with the use of pre-contoured lateral column metaphyseal LCP by triceps-sparing posterolateral approach. Strategies Trauma Limb Reconstr 12:1-9

17. Ko S-H, Cha J-R, Lee CC et al (2017) Minimally invasive plate osteosynthesis using a screw compression method for treatment of humeral shaft fractures. Clin Orthop Surg 9:506-513

18. Lee HM, Kim YS, Kang S et al (2018) Modified anterolateral approach for internal fixation of Holstein-Lewis humeral shaft fractures. J Orthop Sci 23:137-143

19. Matsunaga FT, Tamaoki MJS, Matsumoto MH et al (2017) Minimally invasive osteosynthesis with a bridge plate versus a functional brace for humeral shaft fractures: a randomized controlled trial. J Bone Joint Surg Am 99:583-592

20. Pal JN, Biswas P, Roy A et al (2015) Outcome of humeral shaft fractures treated by functional cast brace. Indian J Orthop 49:408-417

21. Salvador J, Amhaz-Escanlar S, Castillón P et al (2018) Cerclage wiring and intramedullary nailing, a helpful and safe option specially in proximal fractures. A multicentric study. Injury. https:// doi.org/10.1016/j.injury.2018.11.042

22. Seo J-B, Heo K, Yang J-H, Yoo J-S (2019) Clinical outcomes of dual 3.5-mm locking compression plate fixation for humeral shaft fractures: Comparison with single 4.5-mm locking compression plate fixation. J Orthop Surg 27:2309499019839608. https://doi. org/10.1177/2309499019839608

23. Singh AK, Narsaria N, Seth RR, Garg S (2014) Plate osteosynthesis of fractures of the shaft of the humerus: comparison of limited contact dynamic compression plates and locking compression plates. J Orthop Traumatol 15:117-122

24. Wang Q, Hu J, Guan J et al (2018) Proximal third humeral shaft fractures fixed with long helical PHILOS plates in elderly patients: benefit of pre-contouring plates on a 3D-printed model-a retrospective study. J Orthop Surg Res 13:203

25. Yin $\mathrm{P}, \mathrm{Zhang} \mathrm{L}, \mathrm{Mao} \mathrm{Z}$ et al (2014) Comparison of lateral and posterior surgical approach in management of extra-articular distal humeral shaft fractures. Injury 45:1121-1125 
26. Zogaib RK, Morgan S, Belangero PS et al (2014) Minimal invasive ostheosintesis for treatment of diaphiseal transverse humeral shaft fractures. Acta Ortop Bras 22:94-98

27. Shen L, Qin $H, A n Z$ et al (2013) Internal fixation of humeral shaft fractures using minimally invasive plating: comparative study of two implants. Eur J Orthop Surg Traumatol 23:527-534

28. Moher D, Liberati A, Tetzlaff J et al (2009) Preferred reporting items for systematic reviews and meta-analyses: the PRISMA statement. BMJ 339:b2535

29. Coleman BD, Khan KM, Maffulli N et al (2000) Studies of surgical outcome after patellar tendinopathy: clinical significance of methodological deficiencies and guidelines for future studies. Scand J Med Sci Sports 10:2-11

30. An Z, He X, Jiang C, Zhang C (2012) Treatment of middle third humeral shaft fractures: minimal invasive plate osteosynthesis versus expandable nailing. Eur J Orthop Surg Traumatol 22:193-199

31. Apivatthakakul T, Phornphutkul C, Laohapoonrungsee A, Sirirungruangsarn Y (2009) Less invasive plate osteosynthesis in humeral shaft fractures. Oper Orthop Traumatol 21:602-613

32. Brunner A, Thormann S, Babst R (2012) Minimally invasive percutaneous plating of proximal humeral shaft fractures with the Proximal Humerus Internal Locking System (PHILOS). J Shoulder Elbow Surg 21:1056-1063

33. Chao T-C, Chou W-Y, Chung J-C, Hsu C-J (2005) Humeral shaft fractures treated by dynamic compression plates, ender nails and interlocking nails. Int Orthop 29:88-91

34. Concha JM, Sandoval A, Streubel PN (2010) Minimally invasive plate osteosynthesis for humeral shaft fractures: are results reproducible? Int Orthop 34:1297-1305

35. Jawa A, McCarty P, Doornberg J et al (2006) Extra-articular distal-third diaphyseal fractures of the humerus. A comparison of functional bracing and plate fixation. J Bone Joint Surg Am 88:2343-2347

36. Lau TW, Leung F, Chan CF, Chow SP (2007) Minimally invasive plate osteosynthesis in the treatment of proximal humeral fracture. Int Orthop 31:657-664

37. Liebergall M, Jaber S, Laster M et al (1997) Ender nailing of acute humeral shaft fractures in multiple injuries. Injury 28:577-580

38. Lin J, Hou S-M (2003) Locked nailing of severely comminuted or segmental humeral fractures. Clin Orthop Relat Res 406:195-204

39. Spagnolo R, Pace F, Bonalumi M (2010) Minimally invasive plating osteosynthesis technique applied to humeral shaft fractures: the lateral approach. Eur J Orthop Surg Traumatol 20:205-210

40. Yang Q, Wang F, Wang Q et al (2012) Surgical treatment of adult extra-articular distal humeral diaphyseal fractures using an oblique metaphyseal locking compression plate via a posterior approach. Med Princ Pract 21:40-45

41. Arora S, Goel N, Cheema GS et al (2011) A method to localize the radial nerve using the "apex of triceps aponeurosis" as a landmark. Clin Orthop Relat Res 469:2638-2644

42. Li Y, Wang C, Wang M et al (2011) Postoperative malrotation of humeral shaft fracture after plating compared with intramedullary nailing. J Shoulder Elbow Surg 20:947-954

43. Livani B, Belangero WD (2004) Bridging plate osteosynthesis of humeral shaft fractures. Injury 35:587-595

44. Malhan S, Thomas S, Srivastav S et al (2012) Minimally invasive plate osteosynthesis using a locking compression plate for diaphyseal humeral fractures. J Orthop Surg 20:292-296
45. Pehlivan $\mathrm{O}$ (2002) Functional treatment of the distal third humeral shaft fractures. Arch Orthop Trauma Surg 122:390-395

46. Zhiquan A, Bingfang Z, Yeming W et al (2007) Minimally invasive plating osteosynthesis (MIPO) of middle and distal third humeral shaft fractures. J Orthop Trauma 21:628-633

47. Ekholm R, Ponzer S, Törnkvist $\mathrm{H}$ et al (2008) The Holstein-Lewis humeral shaft fracture: aspects of radial nerve injury, primary treatment, and outcome. J Orthop Trauma 22:693-697

48. An Z, Zeng B, He X et al (2010) Plating osteosynthesis of middistal humeral shaft fractures: minimally invasive versus conventional open reduction technique. Int Orthop 34:131-135

49. Habernek H, Schmid L, Orthner E (1992) Initial experiences with the humerus interlocking nail. Unfallchirurgie 18:233-237

50. Veltman ES, Doornberg JN, Eygendaal D, van den Bekerom MPJ (2015) Static progressive versus dynamic splinting for posttraumatic elbow stiffness: a systematic review of 232 patients. Arch Orthop Trauma Surg 135:613-617

51. Iliaens J, Metsemakers W-J, Coppens S et al (2019) Regional anaesthesia for surgical repair of proximal humerus fractures: a systematic review and critical appraisal. Arch Orthop Trauma Surg 139:1731-1741

52. Tsai C-H, Fong Y-C, Chen Y-H et al (2009) The epidemiology of traumatic humeral shaft fractures in Taiwan. Int Orthop 33:463-467

53. Claessen FMAP, Peters RM, Verbeek DO et al (2015) Factors associated with radial nerve palsy after operative treatment of diaphyseal humeral shaft fractures. J Shoulder Elbow Surg 24:e307-e311

54. Liu G-Y, Zhang C-Y, Wu H-W (2012) Comparison of initial nonoperative and operative management of radial nerve palsy associated with acute humeral shaft fractures. Orthopedics 35:702-708

55. Sarmiento A, Zagorski JB, Zych GA et al (2000) Functional bracing for the treatment of fractures of the humeral diaphysis. J Bone Joint Surg Am 82:478-486

56. Schwab TR, Stillhard PF, Schibli S et al (2018) Radial nerve palsy in humeral shaft fractures with internal fixation: analysis of management and outcome. Eur J Trauma Emerg Surg 44:235-243

57. Bodner G, Huber B, Schwabegger A et al (1999) Sonographic detection of radial nerve entrapment within a humerus fracture. $\mathrm{J}$ Ultrasound Med 18:703-706

58. Bodner G, Buchberger W, Schocke M et al (2001) Radial nerve palsy associated with humeral shaft fracture: evaluation with USinitial experience. Radiology 219:811-816

59. Esparza M, Wild JR, Minnock C et al (2019) Ultrasound evaluation of radial nerve palsy associated with humeral shaft fractures to guide operative versus non-operative treatment. Acta Med Acad 48:183-192

60. Cartwright MS, Chloros GD, Walker FO et al (2007) Diagnostic ultrasound for nerve transection. Muscle Nerve 35:796-799

Publisher's Note Springer Nature remains neutral with regard to jurisdictional claims in published maps and institutional affiliations. 